\title{
RESEARCH
}

Open Access

\section{Quantitative evaluation of carotid atherosclerotic vulnerable plaques using in vivo T1 mapping cardiovascular magnetic resonaonce: validation by histology}

Huiyu Qiao', Dongye Li ${ }^{1}$, Jingli Cao ${ }^{3}$, Haikun Qi ${ }^{4}$, Yongjun Han ${ }^{1}$, Hualu Han ${ }^{1}$, Huimin Xu' ${ }^{5}$, Tao Wang ${ }^{6}$, Shuo Chen', Huijun Chen', Yajie Wang ${ }^{7}$ and Xihai Zhao ${ }^{1 *}$

\begin{abstract}
Background: It has been proved that multi-contrast cardiovascular magnetic resonance (CMR) vessel wall imaging could be used to characterize carotid vulnerable plaque components according to the signal intensity on different contrast images. The signal intensity of plaque components is mainly dependent on the values of $\mathrm{T} 1$ and $\mathrm{T} 2$ relaxation. T1 mapping recently showed a potential in identifying plaque components but it is not well validated by histology. This study aimed to validate the usefulness of in vivo T1 mapping in assessing carotid vulnerable plaque components by histology.

Methods: Thirty-four subjects (mean age, $64.0 \pm 8.9$ years; 26 males) with carotid plaques referred to carotid endarterectomy were prospectively enrolled and underwent 3 T CMR imaging from May 2017 to October 2017. The $\mathrm{T} 1$ values of intraplaque hemorrhage (IPH), necrotic core (NC) and loose matrix (LM) which were identified on multi-contrast vessel wall images or histology were measured on in-vivo T1 mapping. The IPHs were divided into two types based on the proportion of the area of fresh hemorrhage on histology. The T1 values of different plaque components were compared using Mann-Whitney $U$ test and the agreement between T1 mapping and histology in identifying and quantifying IPH was analyzed with Cohen's Kappa and intraclass correlation coefficient (ICC).

Results: Of 34 subjects, 19 had histological specimens matched with CMR imaging. The mean T1 values of IPH (651 $\pm 253 \mathrm{~ms}), \mathrm{NC}(1161 \pm 182 \mathrm{~ms})$ and LM (1447 $\pm 310 \mathrm{~ms})$ identified by histology were significantly different. The T1 values of Type $1 \mathrm{IPH}$ were significantly shorter than that of Type $2 \mathrm{IPH}(456 \pm 193 \mathrm{~ms}$ vs. $775 \pm 205 \mathrm{~ms}, p<0.001)$. Moderate to excellent agreement was found in identification (kappa $=0.51, p<0.001$ ), classification (kappa $=0.40$, $p=0.028)$ and segmentation (ICC $=0.816,95 \% \mathrm{Cl} 0.679-0.894)$ of IPHs between T1 mapping and histology.

(Continued on next page)
\end{abstract}

\footnotetext{
* Correspondence: xihaizhao@tsinghua.edu.cn

${ }^{1}$ Center for Biomedical Imaging Research, Department of Biomedical

Engineering, Tsinghua University School of Medicine, Haidian District, Beijing 100084, China

Full list of author information is available at the end of the article
}

C C The Author(s). 2020 Open Access This article is licensed under a Creative Commons Attribution 4.0 International License, which permits use, sharing, adaptation, distribution and reproduction in any medium or format, as long as you give appropriate credit to the original author(s) and the source, provide a link to the Creative Commons licence, and indicate if changes were made. The images or other third party material in this article are included in the article's Creative Commons licence, unless indicated otherwise in a credit line to the material. If material is not included in the article's Creative Commons licence and your intended use is not permitted by statutory regulation or exceeds the permitted use, you will need to obtain permission directly from the copyright holder. To view a copy of this licence, visit http://creativecommons.org/licenses/by/4.0/ The Creative Commons Public Domain Dedication waiver (http://creativecommons.org/publicdomain/zero/1.0/) applies to the data made available in this article, unless otherwise stated in a credit line to the data. 
(Continued from previous page)

Conclusions: The T1 values of carotid plaque components, particularly for intraplaque hemorrhage, are

differentiable, and the stage of intraplaque hemorrhage can be classified according to T1 values, suggesting the potential capability of assessment of vulnerable plaque components by T1 mapping.

Keywords: Carotid artery, Atherosclerosis, Intraplaque hemorrhage, T1 mapping, Magnetic resonance imaging

\section{Background}

It is well evidenced that cerebrovascular vulnerable atherosclerotic plaque is the key etiology of ischemic stroke [1]. Histologically, intraplaque hemorrhage (IPH) and large necrotic core (NC) have been considered as the key features of vulnerable plaques [2]. In addition, investigators found that stage of IPH was associated with cardiovascular events [3]. Therefore, it is important to accurately characterize vulnerable plaque components.

Multi-contrast cardiovascular magnetic resonance (CMR) vessel wall imaging, including time-of-flight (TOF), T1-weighted, and T2-weighted sequences, has become an ideal non-invasive technique to assess carotid plaque components [4]. In multi-contrast CMR imaging techniques, each sequence plays a specific role in the identification of specific plaque component. Pre- and post-contrast T1weighted imaging has been largely used to evaluate $\mathrm{NC}$ with good agreement with histology [5]. The sequence of magnetization prepared rapid gradient echo acquisition (MP-RAGE) has been demonstrated to be the best technique in characterizing IPH among three different T1weighted sequences [6]. It is evidenced that the stage of carotid artery IPH can be distinguished by combining TOF, T1-weighted, and T2-weighted images [7].

Since plaque components are determined according to their signal intensity on different contrast images and CMR signal is mainly dependent on the values of T1 and T2 relaxation, it is possible to distinguish plaque components by quantitative imaging. Recently, quantitative CMR imaging techniques, such as T1 mapping, have been proposed for plaque characterization [8-11]. Although investigators utilized ex-vivo T1 mapping to assess intracranial plaques [8], few studies investigated the in-vivo $\mathrm{T} 1$ values of carotid plaque components. Ota and colleagues measured the in-vivo longitudinal relaxation rates $(\mathrm{R} 1=1 / \mathrm{T} 1)$ of carotid homogenous plaque components which could not be applied to characterization of those plaques with inhomogeneous and complicated components [9]. Subsequently, Qi et al. developed a 3D SNAP with golden angle radial k-space sampling (GOAL-SNAP) sequence for measuring T1 values of vessel wall and this technique showed a potential in identifying IPH and monitoring its progression [10]. However, the capability of GOAL-SNAP sequence in characterizing vulnerable plaques has not been validated by histology.

Here, we hypothesized that different plaque components have different $\mathrm{T} 1$ values and the $\mathrm{T} 1$ values of vessel wall can be used to identify vulnerable plaque features. This study sought to validate the usefulness of in vivo T1 mapping in assessing carotid vulnerable plaque compositional features by histology.

\section{Methods}

\section{Study sample}

Thirty-four subjects (mean age, 64.0 \pm 8.9 years; 26 males) with $50-70 \%$ symptomatic stenosis or $\geq 70 \%$ stenosis in carotid artery referred to carotid endarterectomy (CEA) were prospectively and consecutively enrolled from Peking University Third Hospital during May 2017 to October 2017. All subjects underwent CMR imaging for carotid arteries within 1 week before CEA surgery. The exclusion criteria are: 1) stenting therapy; and 2) contraindications to CMR examination. Clinical information including age, gender, height, weight, blood pressure, hyperlipidemia, hypertension, diabetes, and history of cardiovascular disease were collected from medical records. The study protocol was approved by local institutional review board and written informed consent form was obtained from each subject.

\section{Carotid CMR imaging}

Carotid arteries of all subjects were imaged on $3 \mathrm{~T}$ CMR scanner (Achieva TX, Philips Healthcare, Best, The Netherlands) with a dedicated 8-channel carotid coil. CMR vessel wall imaging including T1-quadruple inversion recovery (QIR), T2-multislice double inversion recovery (MDIR), 3D TOF and MP-RAGE sequences and 3D T1 mapping sequence (GOAL-SNAP) [10] was performed centered to the bifurcation of carotid artery which was the target of CEA surgery. The imaging parameters are presented in Table 1.

\section{CMR image analysis}

The images of GOAL-SNAP were off-line reconstructed to inversion recovery (IR) image series using sliding window and the k-space weighted image contrast (KWIC) methods for T1 fitting [12]. To compare with multicontrast CMR images, the reconstructed 3D GOAL- 
Table 1 CMR vessel wall imaging and GOAL-SNAP imaging parameters

\begin{tabular}{|c|c|c|c|c|c|}
\hline & \multicolumn{4}{|c|}{ Multi-contrast CMR imaging } & \multirow{2}{*}{$\begin{array}{l}\text { T1 Mapping } \\
\text { GOAL-SNAP }\end{array}$} \\
\hline & $\mathrm{T} 1-\mathrm{Q} I \mathrm{R}$ & T2-MDIR & 3D TOF & MP-RAGE & \\
\hline Acquisition & TSE & TSE & FFE & TFE & TFE \\
\hline TR/TE (ms) & $800 / 10$ & $4800 / 50$ & $20 / 5.5$ & $13.2 / 3.2$ & $11.3 / 4.7$ \\
\hline Turbo factor & 10 & 12 & - & 55 & 175 \\
\hline Flip angle (deg) & 90 & 90 & 20 & 15 & 8 \\
\hline $\mathrm{FOV}\left(\mathrm{mm}^{3}\right)$ & \multicolumn{2}{|c|}{$160 \times 160 \times 32$} & \multicolumn{2}{|c|}{$160 \times 160 \times 48$} & $100 \times 100 \times 100$ \\
\hline Resolution $\left(\mathrm{mm}^{3}\right)^{\mathrm{a}}$ & \multicolumn{2}{|c|}{$0.6 \times 0.6 \times 2$} & \multicolumn{2}{|c|}{$0.6 \times 0.6 \times 2$} & $0.8 \times 0.8 \times 0.8$ \\
\hline Orientation & Axial & Axial & Axial & Axial & Axial \\
\hline NSA & 1 & 1 & 1 & 2 & 1 \\
\hline Scan time (min) & 6 & 4 & 2 & 4 & 5 \\
\hline
\end{tabular}

TSE turbo spin echo, FFE fast field echo, TFE turbo field echo, TR repetition time, TE echo time, FOV field of view, NSA number of signal averaged, GOAL-SNAP SNAP with golden angle radial $\mathrm{k}$-space sampling

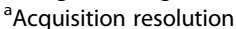

SNAP images were reformatted to $2 \mathrm{~mm}$ axial slices with the same off-center distance and angulation to T1-QIR images using a Philips MR workstation (Extended MR Workspace 2.6.3.1, Philips Medical System). This process can ensure that images with the same number of slices from these two sequences had same geometric position. The reformatted IR images series from GOALSNAP and T1-QIR images were registered for each subject in "CASCADE" software (UW, Seattle, Washington, USA) [13]. The registration between GOAL-SNAP and T1-QIR images was performed by combining the auto registration in software and manual adjustment. The multi-contrast CMR images were reviewed by two radiologists with > 5 years' experience in CMR using custom-designed software of "CASCADE" with consensus. The CMR image quality was assessed with 4-point scale: $1=$ poor; $2=$ marginal; $3=$ good; and $4=$ excellent [14]. Slices with image quality $<2$ were excluded. The boundaries of lumen and outer wall were outlined on each axial slice. The carotid plaque components, including loose matrix (LM), IPH and NC, were identified and their areas were measured on the multi-contrast CMR vessel wall images using the published criteria [4]. The T1 fitting was conducted using the reformatted and registered GOAL-SNAP IR images in the wall regions for each artery. According to the label masks which were obtained from multi-contrast images and the fitted T1 maps, the $\mathrm{T} 1$ values of different plaque components were recorded. The reconstruction and $\mathrm{T} 1$ fitting of GOAL-SNAP images were performed using MATLAB 2014 (MathWorks, Inc. Natick, Massachusetts, USA).

\section{Process and analysis of histological specimen}

After CEA, the excised plaques were fixed in 10\% neutral buffered formalin within $4 \mathrm{~h}$, decalcified in $10 \%$ formic acid, and embedded a bloc in paraffin. The specimen was sectioned with thickness of $10 \mu \mathrm{m}$ every $0.5 \mathrm{~mm}$ and stained with hematoxylin-eosin (H\&E). The histologic sections were matched with the CMR images by experienced pathologist and radiologist according to the position and the shape of lumen, wall, and plaques with the landmarks of bifurcation. The LM, IPH and NC were identified and quantified on each matched histologic section by two experienced pathologists with $>5$ years' experience using ImageJ software and published criteria with consensus $[15,16]$. The IPHs were classified into two types according to the proportion of the area of fresh hemorrhage occupying the total area of IPH on each section: Type $1, \geq 50 \%$; Type $2,<50 \%$. The proportion of the area of fresh hemorrhage occupying the total area of IPH was also recorded on each section. All the histological marks of LM, IPH, NC were mapped onto the registered T1 mapping images and the corresponding T1 values were recorded on each registered slice. Sixteen histological specimens from 16 patients were randomly selected for reproducibility study. The same two pathologists identified plaque components with consensus blinded to the first round of review with one-year time interval to minimize the memory bias. The corresponding marks were mapped onto the registered $\mathrm{T} 1$ mapping images and the T1 values of LM, NC and IPH on these 16 slices were recorded. The classification of IPH was also conducted in the reproducibility study and compared with previous evaluation. The flowchart of analysis of CMR images and histologic sections is shown in Fig. 1a.

\section{Assessment of IPHs, LM and NC with T1 mapping}

The mean T1 values of two types of IPHs which were obtained from above histological analysis were used as cut-off values to identify and classify different types of IPH. For each matched slice, the shortest T1 value was 


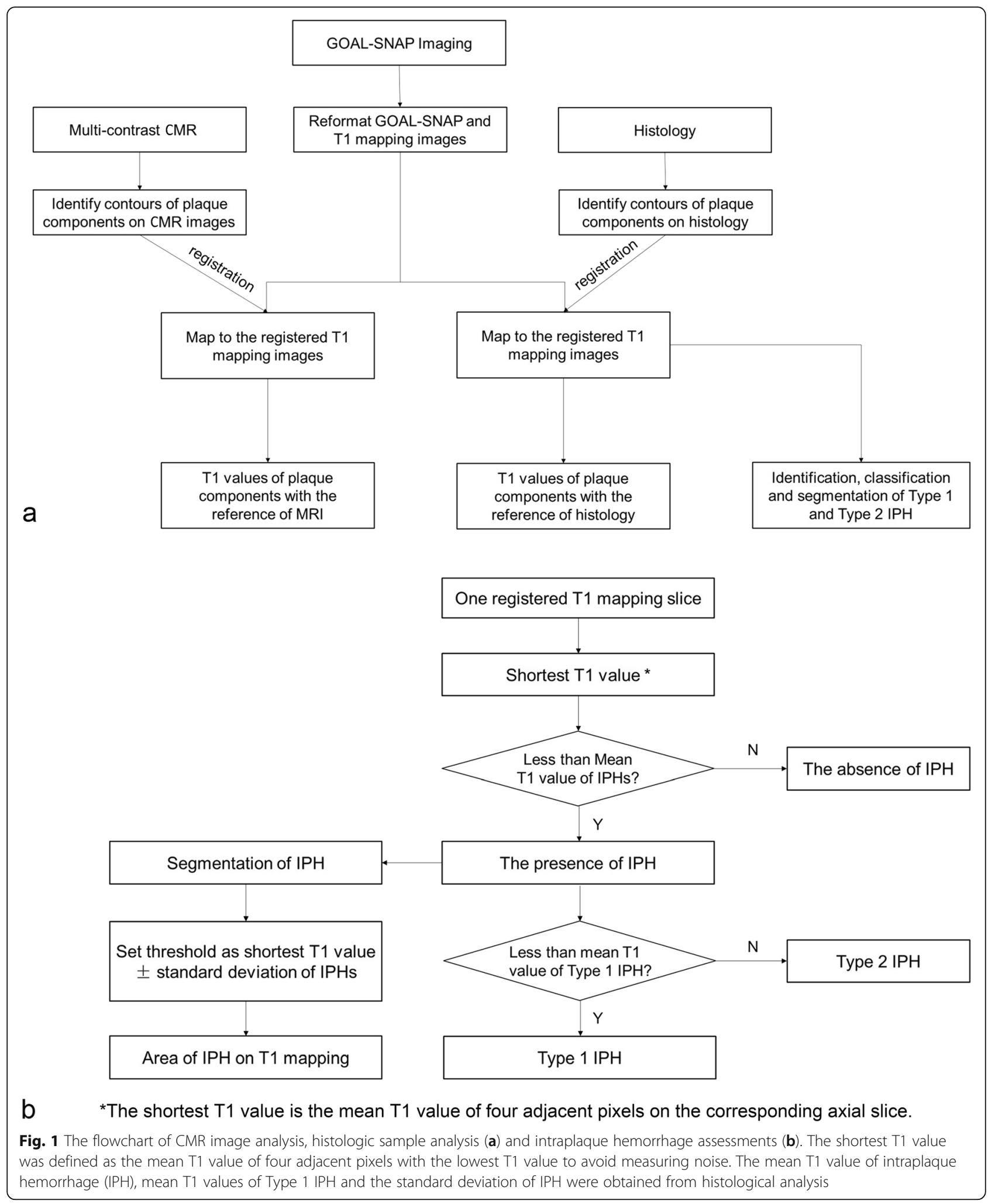

defined as the mean T1 value of four adjacent pixels with the lowest $\mathrm{T} 1$ value to avoid measuring noise. If the shortest $\mathrm{T} 1$ value of the axial slice was shorter than the mean T1 value of both types of IPHs, the presence of
IPH will be identified on the corresponding T1 mapping slice. Among the slices with IPHs, the slices in which the shortest $\mathrm{T} 1$ value was shorter than the mean $\mathrm{T} 1$ value of Type 1 IPHs were classified into Type 1 IPH. In 
contrast, the slices in which the shortest $\mathrm{T} 1$ value was between the mean T1 value of Type 1 IPHs and whole IPHs were classified into Type 2 IPH. The segmentation of IPHs was performed on these slices with IPHs. The threshold-based segmentation of IPHs was also performed. The threshold for the segmentation was set as the sum of the shortest $\mathrm{T} 1$ value and 1 standard deviation of T1 values of IPHs (Fig. 1b).

The identification of LM or NC was based on the hypothesis that the $\mathrm{T} 1$ value of the center of $\mathrm{LM}$ or NC should be around the mean T1 value of the whole LM or NC. The mean T1 value and standard deviation of $\mathrm{T} 1$ value of $\mathrm{LM}$ or $\mathrm{NC}$ were obtained from previous histological analysis. The cut-off T1 value for the identification of LM or NC was set as mean \pm one tenth of standard deviation of $\mathrm{T} 1$ value of $\mathrm{LM}$ or NC. One tenth of standard deviation of T1 value of $\mathrm{LM}$ or $\mathrm{NC}$ was set as a bound to ensure an appropriate size of the connected regions. If there is a region whose mean $\mathrm{T} 1$ value of at least three adjacent pixels was within the threshold range of LM or $\mathrm{NC}$, the corresponding plaque component will be identified.

\section{Statistical analysis}

The continuous variables were described as mean \pm standard deviation and categorical variables were described as percentage. The T1 values of LM, IPHs and $\mathrm{NC}$ identified by multi-contrast images and histology were calculated and compared using one-way ANOVA and Mann-Whitney U tests, respectively. The agreement of $\mathrm{T} 1$ values between two different rounds of review on histological specimen was analyzed using intraclass correlation coefficient (ICC). The accuracy, sensitivity and specificity of above threshold of $\mathrm{T} 1$ value in identifying plaque components were calculated by comparing with the gold standard of histological results using diagnostic test. The agreements in identification of the presence and types of IPHs between T1 mapping and histology were analyzed using Cohen's Kappa test. The correlation between $\mathrm{T} 1$ values and the proportion of the area of fresh hemorrhage occupying the total area of IPH on each section was evaluated using Pearson correlation analysis. The comparison and agreement of the area of IPHs between T1 mapping and histology were performed and determined by using paired $t$ test and ICC analysis, respectively. The area of IPHs measured by histology was adjusted according to the potential shrinkage $(7.8 \%)$ [17]. All statistical analyses were performed using SPSS (v. 16.0 Statistical Package for the Social Sciences, International Business Machines, Inc., Armonk, New York, USA). $P$ values $<0.05$ were considered statistically significant.

\section{Results}

Of 34 recruited patients, 15 (44.1\%) had hypertension, 3 $(8.8 \%)$ had diabetes, 2 (5.9\%) had hyperlipidemia, and 22 (64.7\%) had a history of cardiovascular disease. Of 34 subjects, 1 failed to receive CEA surgery due to perioperative cardiac event and 4 had no intact specimens because of operative procedure. In total, 29 qualified specimens were collected for analysis. The clinical information is detailed in Table 2.

\section{T1 values of plaque components based-on masks of multi-contrast CMR images}

In 34 patients who completed CMR imaging before CEA surgery, 818 slices from 66 carotid arteries with acceptable image quality ( 2 carotid arteries were excluded due to poor image quality) were analyzed. Of 66 carotid plaques from 34 patients, 19 (28.8\%) had NC without IPH, 29 (43.9\%) had IPH, and 36 (54.5\%) had LM on CMR imaging. Of 818 analyzed slices, 285 (34.8\%) had NC without IPH, 161 (19.7\%) had IPH, and 166 (20.3\%) had LM. The T1 values for LM, NC and IPH for each slice are detailed in Fig. 2a. Among all plaque components, IPH showed the shortest T1 value $(907 \pm 354 \mathrm{~ms})$ and LM had the longest T1 value $(1379 \pm 340 \mathrm{~ms})$ (all $p \leq$ 0.001). Typical carotid atherosclerotic plaque images of multi-contrast vessel wall imaging and $\mathrm{T} 1$ mapping are shown in Fig. 3.

\section{T1 values of plaque components validated by histology}

During matching the histological sections of carotid plaque specimens with multicontrast CMR images, 3 specimens were found to have no complicated plaque compositions such as NC and IPH and 7 specimens didn't have the landmark of carotid bifurcation. Therefore, 19 specimens were successfully used for histological validation of T1 mapping. The recruitment of patients and CEA specimens are detailed in Fig. 4.

Table 2 Clinical information of study population $(n=34)$

\begin{tabular}{llc}
\hline & Mean \pm SD or n (\%) & Range \\
\hline Gender, male & $26(76.5)$ & \\
Age, years & $64.0 \pm 8.9$ & $47-81$ \\
Body mass index, $\mathrm{kg} / \mathrm{m}^{2}$ & $24.7 \pm 3.5$ & $18.7-30.8$ \\
Diabetes & $3(8.8)$ & \\
Hypertension & $15(44.1)$ & $105-201$ \\
Systolic blood pressure, $\mathrm{mmHg}$ & $141.5 \pm 19.1$ & $53-93$ \\
Diastolic blood pressure, $\mathrm{mmHg}$ & $74.3 \pm 10.6$ & \\
Hyperlipidemia & $2(5.9)$ & \\
History of cardiovascular disease & $22(64.7)$ & \\
\hline
\end{tabular}


With reference of MRI

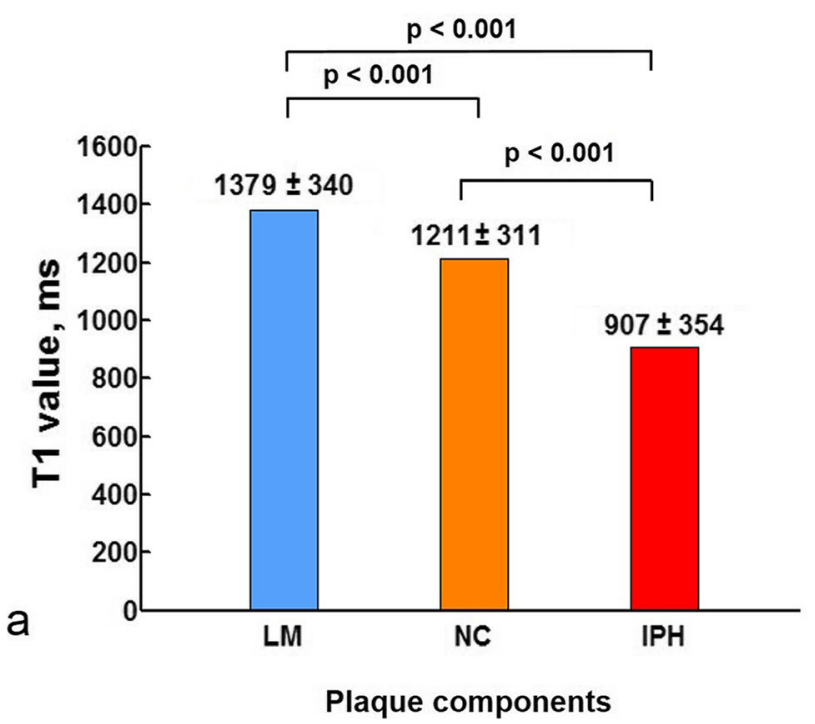

With reference of histology

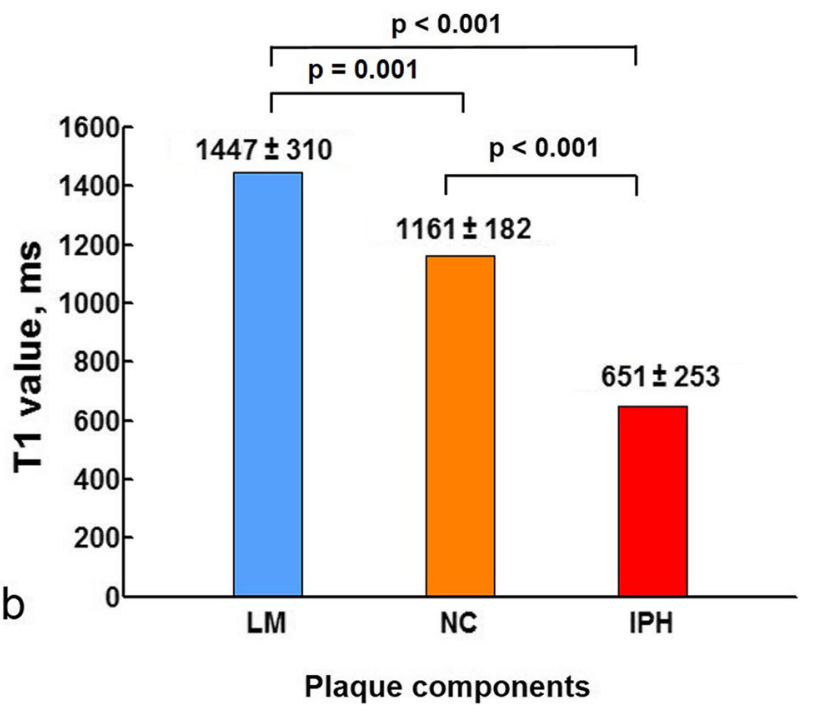

Fig. 2 The plaque components' T1 values calculated with reference of multi-contrast CMR imaging (a) and histology (b). The carotid plaque components included loose matrix (LM), necrotic core (NC) and intraplaque hemorrhage (IPH). IPH appeared the significantly lowest T1 value

In total, 52 slices from 19 CEA specimens of 19 subjects (mean age, $66.2 \pm 7.1$ years; 14 males) were included in the comparison study between histology and T1 mapping. The number of slices of each histological specimen was provided in Table 3 . Of the 19 volunteers with histology data, 12 (63.2\%) had NC, 14 (73.7\%) had IPH, and 14 (73.7\%) had LM. The mean T1 values of IPH, NC and LM for each subject were $685 \pm 226 \mathrm{~ms}, 1171 \pm 164$ $\mathrm{ms}$, and $1382 \pm 301 \mathrm{~ms}$, respectively. Of 52 slices, 25 (48.1\%) had LM, 36 (69.2\%) had IPH, and 24 (46.2\%) had NC on histology. The mean T1 values of IPH, NC, and LM for each slice were $651 \pm 253 \mathrm{~ms}, 1161 \pm 182 \mathrm{~ms}$, and $1447 \pm 310 \mathrm{~ms}$, respectively (all $p \leq 0.001$, Fig. $2 \mathrm{~b}$ ). Of 36 slices with IPH, 14 (38.9\%) and 22 (61.1\%) were classified into Type 1 and Type $2 \mathrm{IPH}$, respectively. The T1 values for Type 1 and Type $2 \mathrm{IPH}$ were $456 \pm 193 \mathrm{~ms}$ and $775 \pm 205 \mathrm{~ms}$, respectively $(p<0.001)$. Of the 16 slices for the reproducibility study, 8 (50.0\%) had NC, 12 (66.7\%) had IPH, and 6 (37.5\%) had LM. Of the 12 slices with IPH, 5 (41.7\%) had Type 1 IPH and 7 (58.3\%) had Type 2 IPH. The identification rate of IPH, NC and LM between two rounds of review by the two pathologists was same. There were excellent agreements of $\mathrm{T} 1$ value measurements (ICC $=0.972,95 \%$ CI $0.937-0.987$ ) and the classification of IPH (ICC $=0.911$, 95\% CI 0.6890.974 ) between two rounds of review for the histological specimens.

Figure 5 represents typical carotid plaque images with LM, IPH and NC on both histologic sections and T1 fitting images.

\section{Assessment of IPHs, LM and NC by T1 mapping validated} by histology

According to the $\mathrm{T} 1$ values of plaque components validated by histology on each slice, the cut-off values for the identification of IPH, NC and LM were set as $\leq 651$ ms (mean T1 value of IPH), 1143-1180 ms (mean T1 value $\pm 1 / 10$ standard deviation of $\mathrm{NC}$ ) and 1416-1478 ms (mean T1 value $\pm 1 / 10$ standard deviation of $\mathrm{LM}$ ), respectively. The cut-off values for the classification of Type 1 IPH and Type 2 IPH were set as $<456 \mathrm{~ms}$ and 456-651 ms, respectively. Moderate agreement was found between T1 mapping and histology in identifying the presence of IPHs (kappa $=0.51, p<0.001)$ and classifying the type of IPHs (kappa $=0.40, p=0.028$ ). The accuracy of T1 mapping in identifying and classifying IPHs was $78.8 \%$ and $70.0 \%$, respectively (Table 4 ). The sensitivity and specificity of T1 mapping in identifying IPHs was $83.3 \%$ and $68.8 \%$, respectively. Strong correlation was found between $\mathrm{T} 1$ values and the proportion of the area of fresh hemorrhage occupying the total area of IPH $(r=0.780, p<0.001$, Fig. 6). Excellent agreement between T1 mapping and histology can be observed in measuring the area of IPHs (ICC $=0.816$, 95\% CI 0.679 0.894). For IPHs detected by both T1 mapping and histological sections, the area of IPHs measured on T1 mapping was larger than that measured on histology, but no significant difference was found $\left(13.7 \pm 8.2 \mathrm{~mm}^{2}\right.$ vs. $11.4 \pm$ $\left.7.2 \mathrm{~mm}^{2}, p=0.080\right)$. The typical images for the segmentation of IPH are shown in Fig. 7. With the reference of histology, the accuracy, sensitivity and specificity of T1 

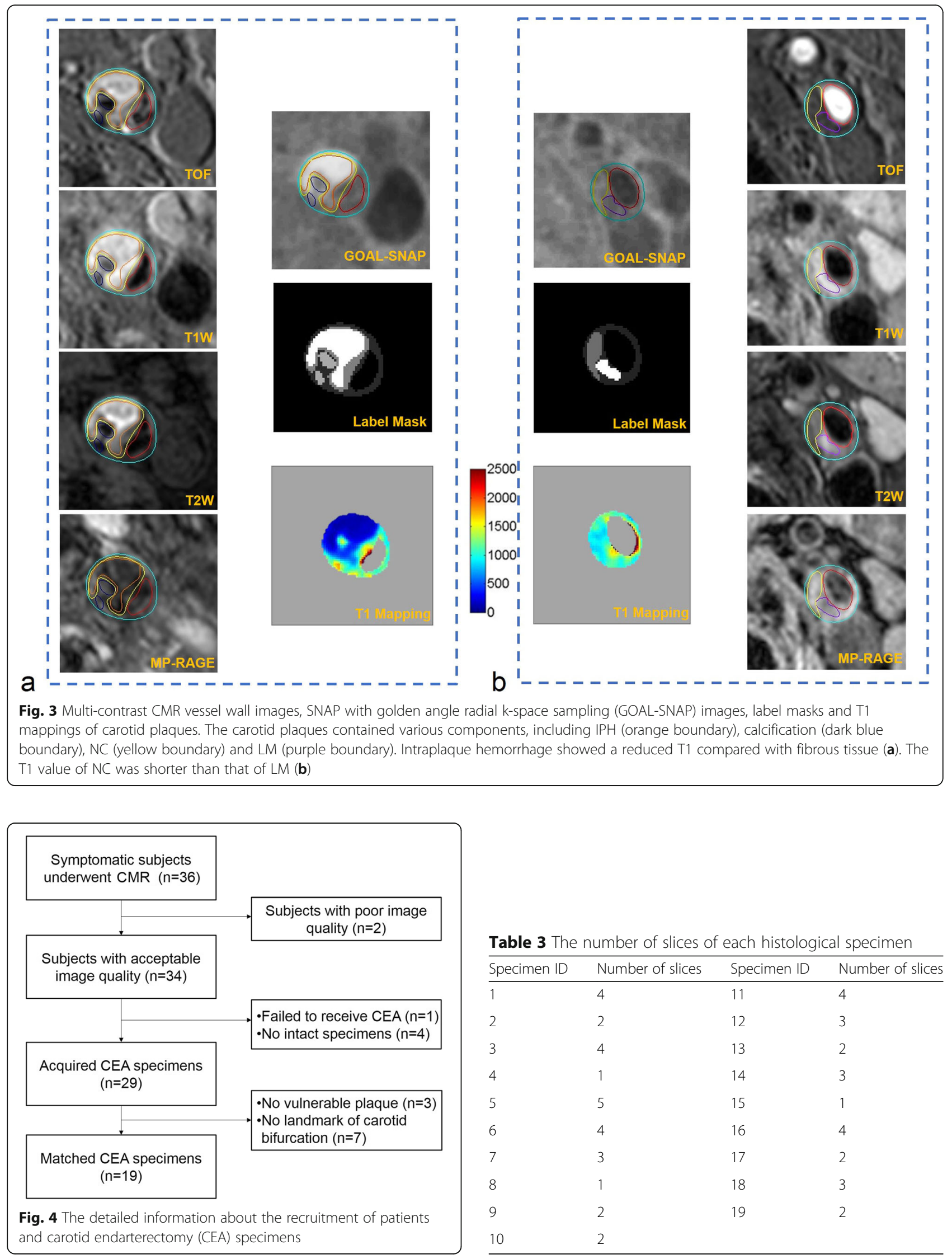

Table $\mathbf{3}$ The number of slices of each histological specimen

\begin{tabular}{llll}
\hline Specimen ID & Number of slices & Specimen ID & Number of slices \\
\hline 1 & 4 & 11 & 4 \\
2 & 2 & 12 & 3 \\
3 & 4 & 13 & 2 \\
4 & 1 & 14 & 3 \\
5 & 5 & 15 & 1 \\
6 & 4 & 16 & 4 \\
7 & 3 & 17 & 2 \\
8 & 1 & 18 & 3 \\
9 & 2 & 19 & 2 \\
10 & 2 & & \\
\hline
\end{tabular}




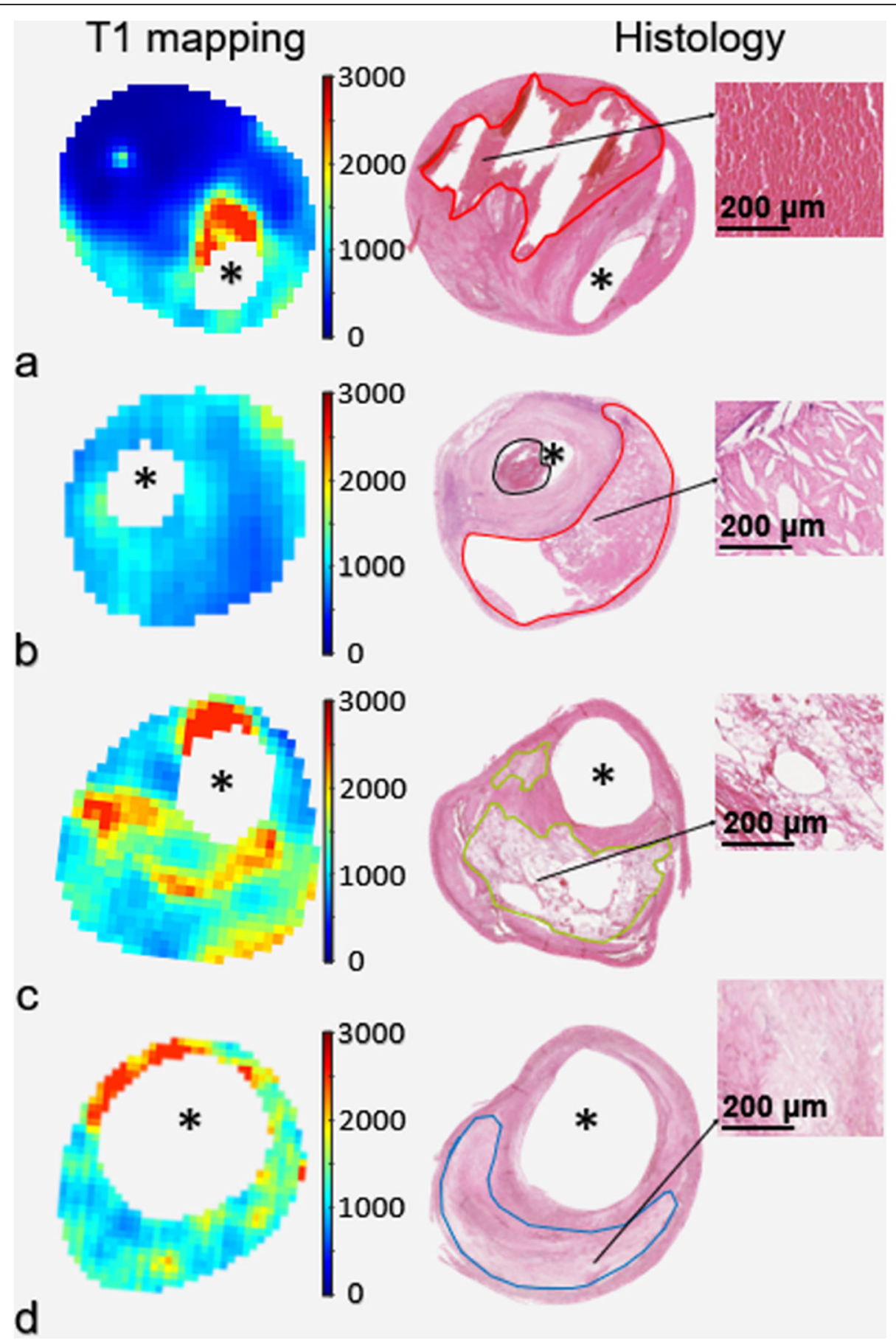

Fig. 5 T1 mapping and H\&E stained histology of carotid vulnerable plaques. Carotid vulnerable plaques with Type 1 intraplaque hemorrhage (red boundary, a), Type 2 IPH (red boundary, b), LM (green boundary, c), and NC (blue boundary, d) are shown, respectively. The black boundary was the mural thrombus inside the lumen

mapping were $53.8 \%, 60.0 \%$ and $48.1 \%$ in identifying LM, and $59.6 \%, 79.2 \%$ and $42.9 \%$ in identifying $\mathrm{NC}$, respectively.

\section{Discussion}

In this study, we calculated the T1 values of carotid atherosclerotic plaque components which were identified on multi-contrast vessel wall imaging or validated by histology. We found that T1 values of IPH, NC and LM were significantly different which suggests the potential capability of T1 mapping in the identification and segmentation of carotid plaque components. Moderate to good agreement was found in the identification, 
Table 4 The agreement between T1 mapping and histology in assessing IPHs

\begin{tabular}{llll}
\hline & & Histology & \\
\cline { 3 - 4 } & & Absence & Presence \\
\hline T1 Mapping & Absence & 11 & 6 \\
& *Presence & 5 & 30 \\
& & Type 1 & Type 2 \\
& Type 1 & 10 & 5 \\
& Type 2 & 4 & 11 \\
\hline
\end{tabular}

*Presence of IPH on T1 mapping was determined when the shortest T1 (the mean T1 value of four adjacent pixels) of the axial slice was shorter than the threshold. IPH: intraplaque hemorrhage

classification and quantitative measurements of IPHs between T1 mapping and histology.

In this study, significant difference in $\mathrm{T} 1$ values was found among NC, IPH and LM validated by multicontrast CMR images or histological specimens. Few previous studies investigated the in-vivo T1 values of carotid plaque components due to the challenges of highresolution vessel wall T1 mapping. Previously, ex-vivo intracranial plaques from 20 cadavers were studied in which the $\mathrm{T} 1$ values of $\mathrm{NC}$, fibrous tissue, fibrous cap, wall and calcification were reported [8]. However, the ex-vivo plaques have different conditions, such as temperature and exchanges of molecules, compared with in-vivo ones. Recently, Ota et al. measured the longitudinal relaxation rates $(\mathrm{R} 1=1 / \mathrm{T} 1)$ of in-vivo carotid plaque components on pixel basis validated by histology [9]. The R1 of NC, IPH and fibrous tissue was reported to be $0.4-1.2 \mathrm{~s}^{-1}$ (T1: 833-2500 ms), $\geq 1.5 \mathrm{~s}^{-1}$ (T1 $\leq 667$ $\mathrm{ms}$ ), and $0.2-0.8 \mathrm{~s}^{-1}$ (T1: $\left.1250-5000 \mathrm{~ms}\right)$, respectively. In contrast, other in-vivo studies at $3 \mathrm{~T}$ reported that the T1 value for the vessel wall, muscle, and IPH was 1195 to $1213 \mathrm{~ms}, 1027$ to $1219 \mathrm{~ms}$, and $371 \pm 93 \mathrm{~ms}$ (the minimum value), respectively $[10,18,19]$. The $\mathrm{T} 1$ value of $\mathrm{NC}$ in the present study was comparable with previous

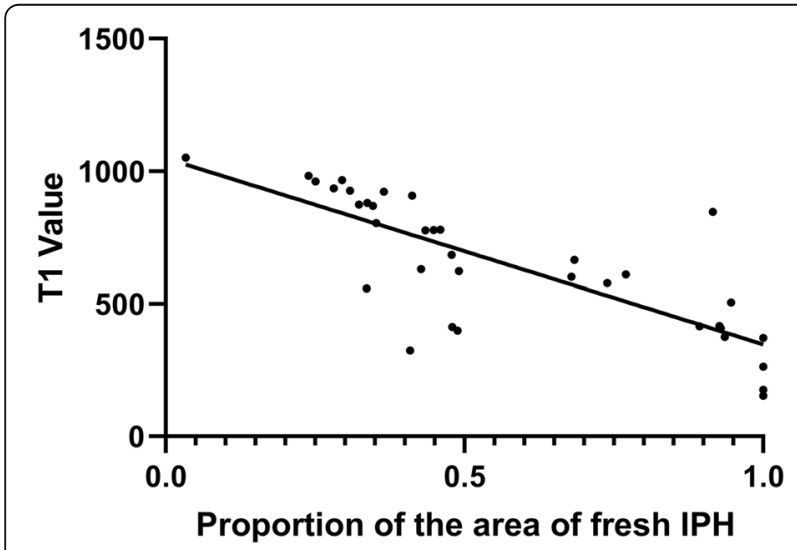

Fig. 6 The regression line between $T 1$ value and the proportion of the area of fresh hemorrhage studies. In addition, the T1 value of IPHs which were identified on multi-contrast CMR images and histology was $907 \pm 354 \mathrm{~ms}$ and $651 \pm 253 \mathrm{~ms}$, respectively. This difference might be caused by a larger proportion of old IPH which showed a longer T1 value than fresh IPH in multi-contrast imaging samples than that in histological specimens $(66.1 \%)$. The difference might be contributed by the mismatched resolution between CMR imaging and histology. The IPHs identified on multi-contrast images might include part of NC which would cause a longer $\mathrm{T} 1$ value. The reported $\mathrm{T} 1$ values of $\mathrm{LM}$ or $\mathrm{NC}$ identified by multi-contrast imaging and histology were similar. Additional value of the present study was that the in-vivo $\mathrm{T} 1$ value of $\mathrm{LM}$ on $3 \mathrm{~T}$ validated by histology was firstly reported. The differentiable $\mathrm{T} 1$ values of carotid plaque components suggest the potential of automatic segmentation of plaque components using T1 mapping.

T1 mapping was found to have a moderate agreement with histology in identification and classification of carotid IPHs. In the present study, the IPHs were divided into two types according to the percentage of fresh hemorrhage on histology. We found that Type $1 \mathrm{IPH}$ showed significantly shorter T1 value than Type $2 \mathrm{IPH}$ and $\mathrm{T} 1$ values had strong correlation with the proportion of area of fresh hemorrhage occupying the total area of IPH, indicating that lower T1 values signify fresher hemorrhage which represents a higher risk of carotid plaque. Histologically, fresh IPH was mainly consisted of intact red blood cells, polymorphonuclear cells, lymphocytes, and scattered macrophages [7]. The intact red blood cell with intracellular methemoglobin in fresh IPH might shorten the T1 values. In addition, a threshold segmentation was also utilized to quantify carotid IPH and good agreement was found in measuring the area of IPHs between T1 mapping and histology in the present study. The threshold segmentation is appealing because it can be used across different centers. In the present study, T1 mapping images had a low accuracy and specificity in identification of NC and LM which might be caused by the overlaps of their $\mathrm{T} 1$ values. The NC has complex components including cholesterol crystal, debris of apoptotic cells and particles of calcium which are attributed to wide range of $\mathrm{T} 1$ value. LM shows a long $\mathrm{T} 1$ value which might be underestimated by the GOAL-SNAP imaging due to the intrinsic limitation of this technique for the long $\mathrm{T} 1$ value validated by spin-echo sequence [10]. Therefore, it was challenging to accurately identify and segment the NC and LM using T1 mapping. In addition, the identification and segmentation of plaque components using T1 mapping were based on the boundaries of arterial wall which dominantly determined by T1-QIR images of multicontrast vessel wall imaging. Because the wall 


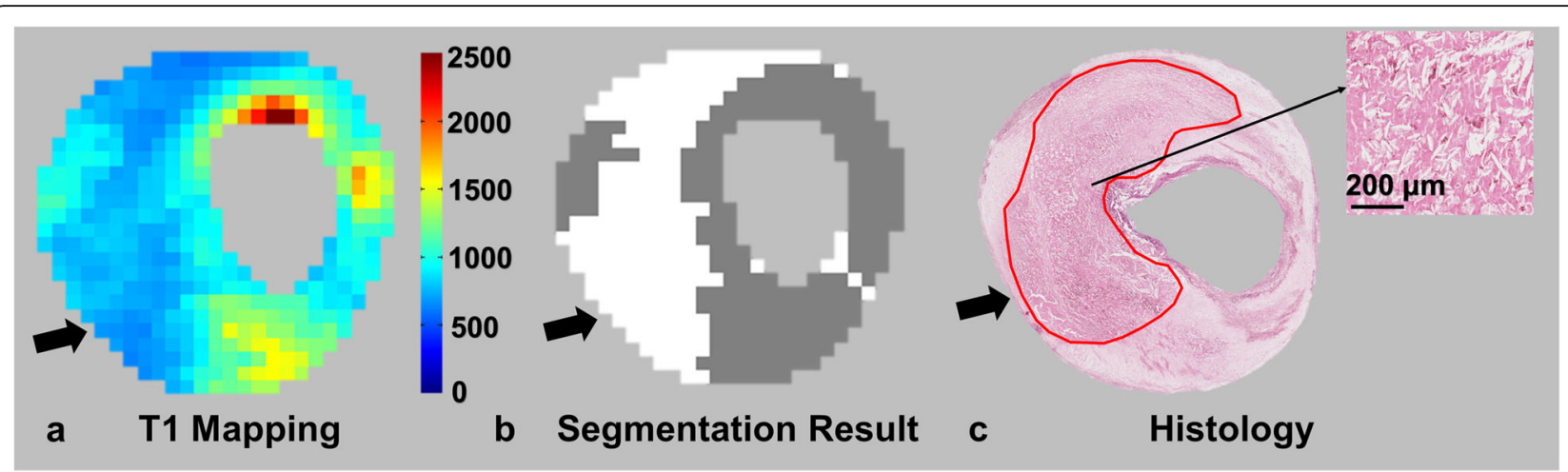

Fig. 7 Typical images for the segmentation of IPHs (arrows). The areas of IPHs on T1 mapping and histology were $17.8 \mathrm{~mm}^{2}$ and $16.0 \mathrm{~mm}^{2}$, respectively

boundaries on T1 mapping images were not clear, the T1 mapping images need to be combined with at least one black blood $\mathrm{T} 1$ weighted sequence to identify plaque components.

There are several limitations in this study. First, the registration of CMR images and histology might be influenced by the mismatched resolution. The histological sections were excised every $0.5 \mathrm{~mm}$ with $10 \mu \mathrm{m}$ thickness. In contrast, the thickness of CMR images was $2 \mathrm{~mm}$. High-resolution CMR vessel wall imaging along with decreased partial volume effects is warranted in future studies. Second, fibrous cap, as a key feature of vulnerable plaques, was not evaluated due to the limited spatial resolution. A previous histological study showed that the minimum thickness of thin fibrous cap was $200 \mu \mathrm{m}$ [20], suggesting that a higher resolution CMR imaging will be required for assessment of fibrous cap. Third, calcification was not evaluated in this study due to the low signal-to-noise ratio of calcification and reliable $\mathrm{T} 1$ cannot be fitted. Qi et al. set the value of T1 and T2 of calcification as zero to realize the segmentation of calcification [18]. Fourth, fibrous tissue was not evaluated in this study. Masson Trichrome stain for the assessment of fibrous tissue is warranted in the future. Fifth, the sample size of our study was relatively small and most of results were from slice-level analysis which might cause over/under representation of some subjects and introduce bias. A larger sample size with the consideration of inter-subject variability was warranted for the validation of $\mathrm{T} 1$ mapping in identifying plaque components with enough training and test datasets. Finally, histological sections were ex-vivo samples in which plaque components might have morphological changes to some extent. Gamble et al. reported that about $7.8 \%$ of shrinkage was determined in whole exvivo specimens [17]. However, the shrinkage of each plaque component has not been investigated. Furthermore, the ex-vivo specimens might have different shape compared with the in-vivo sections.

\section{Conclusions}

CMR T1 values of carotid plaque components, particularly for IPH, are differentiable, and the stage of IPH can be classified according to $\mathrm{T} 1$ values, suggesting the potential capability of assessment of vulnerable plaque components by $\mathrm{T} 1$ mapping.

\section{Abbreviations \\ CEA: Carotid endarterectomy; CMR: Cardiovascular magnetic resonance; GOAL-SNAP: SNAP with golden angle radial k-space sampling sequence; IPH: Intraplaque hemorrhage; IR: Inversion recovery; KWIC: K-space weighted image contrast; LM: Loose matrix; MDIR: Multislice double inversion recovery; MP-RAGE: Magnetization prepared rapid gradient echo acquisition; NC: Necrotic core; QIR: Quadruple inversion recovery; SNAP: Simultaneous non-contrast angiography and intraplaque hemorrhage; T1w: T1 weighted; T2w: T2 weighted; TOF: Time of flight}

\section{Acknowledgments}

None.

\section{Authors' contributions}

Q.H. acquired the CMR data, performed the statistical analysis and drafted manuscript. L.D. analyzed the CMR data. C.J. and W.Y. involved in the histological analysis. Q.H. and C.H. provided CMR technique support. H.Y. processed the histological specimens. H.H. and X.H. acquired the CMR data. W.T. recruited the patients. C.S., Q.H. and Z.X. conceived and designed the experiments. Z.X. conceived the overall study, handled funding and supervision, and drafted the manuscript. All authors read and approved the final manuscript.

\section{Funding}

This study was funded by grants of National Natural Science Foundation of China (81771825), Beijing Municipal Science and Technology Commission (D171100003017003), and Ministry of Science and Technology China (2017YFC1307904).

\section{Availability of data and materials}

The data that support the findings of this study are available on request from the corresponding author [Z.X.]. The data are not publicly available due to them containing information that could compromise research participant privacy/consent. 


\section{Ethics approval and consent to participate}

The study protocol was approved by institutional review board of Tsinghua University School of Medicine and the written consent forms were obtained from all the subjects prior to the initiation of this study.

\section{Consent for publication}

Not applicable.

\section{Competing interests}

The authors declare that they have no competing interests.

\section{Author details}

${ }^{1}$ Center for Biomedical Imaging Research, Department of Biomedical Engineering, Tsinghua University School of Medicine, Haidian District, Beijing 100084, China. ${ }^{2}$ Department of Radiology, Sun Yat-sen Memorial hospital, Sun Yat-sen University, Guangzhou, China. ${ }^{3}$ China National Clinical Research Center for Neurological Diseases, Beijing Tiantan Hospital, Capital Medical University, Beijing, China. ${ }^{4}$ School of Biomedical Engineering and Imaging Sciences, King's College London, London, UK. ${ }^{5}$ Department of Radiology, Peking University Third Hospital, Beijing, China. ${ }^{6}$ Department of Neurosurgery, Peking University Third Hospital, Beijing, China. ${ }^{7}$ Department of Clinical Laboratory, Beijing Ditan Hospital, Capital Medical University, Beijing, China.

Received: 13 November 2019 Accepted: 7 April 2020

Published online: 21 May 2020

\section{References}

1. Zavodni AE, Wasserman BA, McClelland RL, Gomes AS, Folsom AR, Polak JF, Lima JA, Bluemke DA. Carotid artery plaque morphology and composition in relation to incident cardiovascular events: the multi-ethnic study of atherosclerosis (MESA). Radiology. 2014;271:381-9. https://doi.org/10.1148/ radiol. 14131020.

2. Saam T, Hatsukami TS, Takaya N, Chu B, Underhill H, Kerwin WS, Cai J, Ferguson MS, Yuan C. The vulnerable, or high-risk, atherosclerotic plaque: noninvasive MR imaging for characterization and assessment. Radiology. 2007;244:64-77. https://doi.org/10.1148/radiol.2441051769.

3. Cui Y, Qiao H, Ma L, Lu M, Yang J, Yao G, Cai J, Zhao X. Association of age and size of carotid artery intraplaque hemorrhage and minor fibrous cap disruption: a high resolution magnetic resonance imaging study. J Atheroscler Thromb. 2018;25:1222-30. https://doi.org/10.5551/jat.43679.

4. Cai JM, Hatsukami TS, Ferguson MS, Small R, Polissar NL, Yuan C. Classification of human carotid atherosclerotic lesions with in vivo multicontrast magnetic resonance imaging. Circulation. 2002;106:1368-73. https://doi.org/10.1161/01.CIR.0000028591.44554.F9.

5. Cai J, Hatsukami TS, Ferguson MS, Kerwin WS, Saam T, Chu B, Takaya N, Polissar NL, Yuan C. In vivo quantitative measurement of intact fibrous cap and lipid-rich necrotic core size in atherosclerotic carotid plaque: comparison of high-resolution, contrast-enhanced magnetic resonance imaging and histology. Circulation. 2005;112:3437-44. https://doi.org/10. 1161/CIRCULATIONAHA. 104.528174.

6. Ota H, Yarnykh VL, Ferguson MS, Underhill HR, Demarco JK, Zhu DC, Oikawa M, Dong L, Zhao X, Collar A, Hatsukami TS, Yuan C. Carotid intraplaque hemorrhage imaging at 3.0-T MR imaging: comparison of the diagnostic performance of three T1-weighted sequences. Radiology. 2010;254:551-63. https://doi.org/10.1148/radiol.09090535.

7. Chu B, Kampschulte A, Ferguson MS, Kerwin WS, Yarnykh VL, O'Brien KD, Polissar NL, Hatsukami TS, Yuan C. Hemorrhage in the atherosclerotic carotid plaque: a high-resolution MRI study. Stroke. 2004;35:1079-84. https:// doi.org/10.1161/01.STR.0000125856.25309.86.

8. Jiang Y, Zhu C, Peng W, Degnan AJ, Chen L, Wang X, Liu Q, Wang Y, Xiang Z, Teng Z, Saloner D, Lu J. Ex-vivo imaging and plaque type classification of intracranial atherosclerotic plaque using high resolution MRI. Atherosclerosis. 2016;249:10-6. https://doi.org/10.1016/j.atherosclerosis.2016.03.033.

9. Ota H, Tamura H, Itabashi R, Yazawa Y, Nakamura Y, Hisamatsu K, Takamatsu M, Endo H, Niizuma K, Enomoto Y, Nagasaka T, Kajita K, Watanabe M, Yoshimura S, Yuan C. Quantitative characterization of carotid plaque components using MR apparent diffusion coefficients and longitudinal relaxation rates at 3T: a comparison with histology. J Magn Reson Imaging. 2018:48:1657-67. https://doi.org/10.1002/jmri.26216.
10. Qi H, Sun J, Qiao H, Chen S, Zhou Z, Pan X, Wang Y, Zhao X, Li R, Yuan C, Chen $\mathrm{H}$. Carotid intraplaque hemorrhage imaging with quantitative vessel wall T1 mapping: technical development and initial experience. Radiology. 2018;287:276-84. https://doi.org/10.1148/radiol.2017170526.

11. Eto A, Kinoshita Y, Matsumoto Y, Kiyomi F, Iko M, Nii K, Tsutsumi M, Sakamoto K, Aikawa H, Kazekawa K. Relationship between the carotid plaque $\mathrm{T1}$ relaxation time and the plaque-to-muscle signal intensity ratio on black-blood magnetic resonance imaging scans. J Stroke Cerebrovasc Dis. 2016;25:2580-4. https://doi.org/10.1016/j.jstrokecerebrovasdis.

12. Neumann D, Breuer FA, Völker M, Brandt T, Griswold MA, Jakob PM, Blaimer $M$. Reducing contrast contamination in radial turbo-spin-echo acquisitions by combining a narrow-band KWIC filter with parallel imaging. Magn Reson Med. 2014;72:1680-6. https://doi.org/10.1002/mrm.25081.

13. Kerwin W, Xu D, Liu F, Saam T, Underhill H, Takaya N, Chu B, Hatsukami T, Yuan C. Magnetic resonance imaging of carotid atherosclerosis: plaque analysis. Top Magn Reson Imaging. 2007;18:371-8. https://doi.org/10.1097/ rmr.0b013e3181598d9d.

14. Yuan C, Mitsumori LM, Ferguson MS, Polissar NL, Echelard D, Ortiz G, Small R, Davies JW, Kerwin WS, Hatsukami TS. In vivo accuracy of multispectral magnetic resonance imaging for identifying lipid-rich necrotic cores and intraplaque hemorrhage in advanced human carotid plaques. Circulation. 2001;104:2051-6. https://doi.org/10.1161/hc4201.097839.

15. Schneider CA, Rasband WS, Eliceiri KW. NIH image to ImageJ: 25 years of image analysis. Nat Methods. 2012;9:671-5. https://doi.org/10.1038/nmeth.2089.

16. Stary HC, Chandler AB, Dinsmore RE, Fuster V, Glagov S, Insull W Jr, Rosenfeld ME, Schwartz CJ, Wagner WD, Wissler RW. A definition of advanced types of atherosclerotic lesions and a histological classification of atherosclerosis. A report from the committee on vascular lesions of the council on arteriosclerosis, American Heart Association. Circulation. 1995;92: 1355-74. https://doi.org/10.1161/01.CIR.92.5.1355.

17. Gamble G, Beaumont B, Smith H, Zorn J, Sanders G, Merrilees M, MacMahon $S$, Sharpe N. B-mode ultrasound images of the carotid artery wall: correlation of ultrasound with histological measurements. Atherosclerosis. 1993;102:163-73. https://doi.org/10.1016/0021-9150(93)90158-Q.

18. Qi H, Sun J, Qiao H, Zhao X, Guo R, Balu N, Yuan C, Chen H. Simultaneous T1 and T2 mapping of the carotid plaque (SIMPLE) with T2 and inversion recovery prepared 3D radial imaging. Magn Reson Med. 2018;80:2598-608. https://doi.org/10.1002/mrm.27361.

19. Wang N, Christodoulou AG, Xie Y, Wang Z, Deng Z, Zhou B, Lee S, Fan Z, Chang H, Yu W, Li D. Quantitative 3D dynamic contrast-enhanced (DCE) MR imaging of carotid vessel wall by fast T1 mapping using multitasking. Magn Reson Med. 2019;81:2302-14. https://doi.org/10.1002/mrm.27553.

20. Ota H, Yu W, Underhill HR, Oikawa M, Dong L, Zhao X, Polissar NL, Neradilek B, Gao T, Zhang Z, Yan Z, Guo M, Zhang Z, Hatsukami TS, Yuan C. Hemorrhage and large lipid-rich necrotic cores are independently associated with thin or ruptured fibrous caps an in vivo 3T MRI study. Arterioscler Thromb Vasc Biol. 2009;29:1696-701. https://doi.org/10.1161/ ATVBAHA.109.192179.

\section{Publisher's Note}

Springer Nature remains neutral with regard to jurisdictional claims in published maps and institutional affiliations.

Ready to submit your research? Choose BMC and benefit from:

- fast, convenient online submission

- thorough peer review by experienced researchers in your field

- rapid publication on acceptance

- support for research data, including large and complex data types

- gold Open Access which fosters wider collaboration and increased citations

- maximum visibility for your research: over $100 \mathrm{M}$ website views per year

At BMC, research is always in progress.

Learn more biomedcentral.com/submissions 